\title{
Comportamento de variáveis fisiológicas durante a aula de hidroginástica com mulheres
}

\author{
Behavior of physiological variables during a water gymnastics \\ class in women
}

\author{
Mabel Micheline Olkoski \\ Dalila Tosset \\ Marcelo Diederichs Wentz \\ Silvana Corrêa Matheus
}

1. Universidade Federal de Santa Maria. Centro de Educação Física e Desportos. Laboratório de Cineantropometria. Santa Maria, RS, Brasil.

2. Universidade Federal de Santa Maria. Departamento de Métodos e Técnicas Desportivas. Laboratório de Cineantropometria. Santa Maria, RS, Brasil.

Recebido em 11/03/08 Revisado em 12/06/08 Aprovado em 19/06/09
Resumo - O objetivo deste estudo foi analisar o comportamento de variáveis fisiológicas e do Índice de Percepção de Esforço (IPE) durante os diferentes momentos da aula de hidroginástica. Foram estudadas 17 mulheres, universitárias (idade 23,06 22,05 anos) investigadas em duas etapas: 1$)$ teste de esforço máximo na esteira ( $\mathrm{VO}_{2}$ pico e FCmáx); e 2) avaliação da composição corporal e realização da aula de hidroginástica ( $\mathrm{FC}, \mathrm{VO}_{2}$, [lac] e IPE). Utilizaram-se a estatística descritiva e ANOVA medidas repetidas, com o teste post hoc Student-Newman-Keuls $(\mathrm{p}<0,05)$. Os resultados mostraram que tanto a FC, quanto o $\mathrm{VO}_{2}$ apresentaram valores estatisticamente diferentes $(\mathrm{p}=0,000)$ nos três períodos da aula. Os valores de lactato sanguíneo apresentaram-se diferentes estatisticamente $(\mathrm{p}=0,001)$, somente quando foram comparados os valores obtidos no período inicial $(1,55 \mathrm{mM})$ e principal $(3,58$ mM). O IPE médio foi de 11 e o gasto calórico total foi de 262,10 kcal. Conclui-se que as variáveis fisiológicas estudadas e o IPE apresentam uma variação significativa em função das três fases da aula de hidroginástica. Além disso, as intensidades de esforço (FC, $\mathrm{VO}_{2}$, [lac]) obtidas no período principal estão dentro dos padrões estabelecidos pela literatura para exercícios aeróbios. Dessa forma, pode-se supor que a prática regular de aulas de hidroginástica com esta estrutura, pode causar uma melhora da condição física de mulheres adultas jovens.

Palavras-chave: Hidroginástica; Consumo de oxigênio; Freqüência cardíaca; Índice de percepção de esforço; Lactato.

Abstract - The aim of this study was to analyze the behavior of physiological variables and rating of perceived exertion (RPE) during the different phases of a water gymnastics class. Seventeen female university students (age: $23 \pm 3.5$ years) were evaluated in two steps: 1) incremental treadmill test (peak $\mathrm{VO}_{2}$ and $\mathrm{HR}_{\text {max }}$ ); 2) assessment of body composition and achievement in the water gymnastics class ( $\mathrm{HR}, \mathrm{VO}_{2}$, [lac], and RPE). Descriptive statistics and repeated measures ANOVA with the post hoc Student-Newman-Keuls were used $(p<0.05)$. The results showed that both $\mathrm{HR}$ and $\mathrm{VO}_{2}$ differed significantly $(p=0.000)$ between the three phases of the class. Blood lactate only differed significantly $(p=0.001)$ between the early $(1.55 \mathrm{mM})$ and the main phase $(3.58 \mathrm{mM})$. The mean RPE was 11 and total calorie expenditure was $262.10 \mathrm{kcal}$. In conclusion, the physiological variables studied and RPE vary significantly according to the phase of the water gymnastics class. In addition, the intensity of effort ( $\mathrm{HR}, \mathrm{VO}_{2}$ and [lac]) obtained for the main phase is within the parameters established in the literature for aerobic exercise. Thus, regular water gymnastics classes with this structure may improve the physical condition of young adult women.

Key words: Water gymnastics; Oxygen consumption; Heart rate; Rating of perceived exertion; Lactate. 


\section{INTRODUÇÃO}

A hidroginástica é uma atividade alternativa de condicionamento físico, constituída de exercícios aquáticos específicos, baseados no aproveitamento da resistência da água como sobrecarga e que tem sido amplamente difundida em clubes, academias e clínicas e tem sido utilizada com diversas finalidades, incluindo o lazer, a prevenção e a recuperação de lesões ${ }^{1,2}$.

Sendo a água um novo ambiente utilizado, devem-se considerar as alterações orgânicas provocadas pela imersão no meio líquido ${ }^{3}$, em função da pressão hidrostática ${ }^{4}$, e da termodinâmica ${ }^{5}$, que podem variar de acordo com a profundidade de imersão ${ }^{1}$ e os diferentes exercícios ${ }^{6}$. Além disso, não há consenso sobre o comportamento da concentração de lactato sanguíneo [Lac], FC, consumo de oxigênio $\left(\mathrm{VO}_{2}\right)^{1,7}$, índice de percepção de esforço (IPE) e gasto calórico (Gcal) durante os exercícios no meio líquido.

Os estudos existentes com hidroginástica apresentam metodologias variadas e diferentes resultados quando se referem ao comportamento da FC e do $\mathrm{VO}_{2}$. São realizados com exercícios isolados, comparando velocidades de movimentos distintas, os meios aquático e terrestre ${ }^{1,7,8}$ e diferentes exercícios $^{1,2,6-8}$. Com relação ao comportamento da [Lac], as investigações ${ }^{1,2}$ utilizam exercícios isolados e diferentes profundidades de água.

O Gcal não foi encontrado em nenhum estudo com hidroginástica. A literatura ${ }^{9-12}$ referente às aulas de ginástica aeróbica demonstraram valores semelhantes (em torno de 5,7 kcal.min).

Apesar do IPE ser um índice utilizado para a prescrição de exercícios de caminhada na água e em terra $^{13}$, nenhum estudo descreve o comportamento desta variável durante aulas de hidroginástica, apenas durante exercícios isolados de hidroginástica realizados a uma cadência fixa ${ }^{6}$.

Em função da diversidade metodológica evidenciada, da ausência de estudos envolvendo aulas de hidroginástica e com o intuito de subsidiar o planejamento e a prescrição de aulas de hidroginástica, têm-se como objetivo analisar o comportamento da FC, do $\mathrm{VO}_{2}$, da [Lac], do IPE e do Gcal durante uma aula de hidroginástica.

\section{PROCEDIMENTOS METODOLÓGICOS}

Foram investigadas 17 universitárias (23,06 2,05 anos), praticantes de hidroginástica há, no mínimo, seis meses e que não tomavam medicação que pudesse influenciar os resultados. Foram investi- gadas todas as universitárias que se apresentaram voluntariamente após convite realizado durante as aulas, enquadraram-se nos critérios de inclusão e assinaram o Termo de Consentimento Livre e Esclarecido aprovado pelo Comitê de Ética em Pesquisa da Universidade Federal de Santa Maria (n 0070.0.243.000-06).

A coleta de dados aconteceu em duas etapas:

1) teste progressivo de esforço máximo (TPEM) na esteira ergométrica (Inbramed-ATL 10200). Utilizou-se o protocolo de Mader ${ }^{14}$ modificado para mulheres, com os seguintes critérios para interrupção: a) exaustão voluntária; ou b) presença de um dos seguintes itens: estabilização da FC (obtida a cada minuto; Polar ${ }^{\circledR}$-Accurex Plus); taxa de troca respiratória $>1,15$; IPE $>17$ (escala de Borg de 6-20 apresentada 30 segundos antes de finalizar cada estágio); e platô no $\mathrm{VO}_{2}$. As variáveis ventilatórias foram obtidas a cada 20 segundos (analisador de gases TEEM 100 AeroSport Inc.).

O intervalo entre as duas etapas foi de no mínimo 48 horas. Cada sujeito teve sua avaliação agendada no mesmo horário e turno do dia.

2) avaliação da composição corporal (CC) e realização da aula de hidroginástica. Para avaliação da CC, realizada na fase pós-menstrual $\left(6^{\circ}-10^{\circ}\right.$ dia), utilizou-se a estatura (estadiômetro de madeira, precisão 0,5cm), massa corporal (balança Welmy, precisão 0,100kg) e dobras cutâneas (compasso Cescorf ${ }^{\mathrm{TM}}$, precisão 0,1mm): axial média, suprailíaca, coxa (terço superior) e panturrilha medial. A densidade corporal (Equação de Petroski ${ }^{15}$ ) foi utilizada para obtenção do percentual de gordura corporal (\%GC) através da fórmula de Siri, caracterizando o grupo.

A aula de hidroginástica foi realizada individualmente, em um tanque com 1,71m de diâmetro e $1,50 \mathrm{~m}$ de profundidade. A temperatura foi mantida entre 32 e $33^{\circ} \mathrm{C}$ (temperatura termoneutra para exercícios no meio líquido ${ }^{16}$ ) e os sujeitos imersos até a altura do processo xifóide ${ }^{6,7}$. Utilizou-se a mesma sequência de músicas (136bpm, compact disc Toshiba) para todos os sujeitos.

A estrutura da aula baseou-se em um estudo sobre hidroginástica realizado por Moraes et al. ${ }^{2}$, que indicou serem necessários 140 segundos de exercícios submáximos para as variáveis fisiológicas entrarem em "steady state". Dessa forma, a cada 140 segundos, trabalhou-se um grupamento muscular e a cada 70 segundos, foi modificado o movimento de 
membros superiores (MMS), para evitar a monotonia. $\mathrm{O}$ mesmo movimento de membros inferiores (MMI) foi mantido durante 350 segundos; assim, para cada MMI, foram utilizados 5 MMS.

A aula foi constituída de: Período inicial (5 minutos): alongamentos (deltóide, peitorais, dorsais quadríceps e panturrilha) e aquecimentos articulares (cervical, ombros, cotovelos, punhos e quadril); Período principal (35 minutos): exercícios aeróbios com MMS realizados concomitantemente com MMI, totalizando 30 exercícios; e Período final (5 minutos): alongamentos (deltóide, bíceps, tríceps, peitorais, dorsais, quadríceps, isquiotibiais e panturrilha).

O período principal foi constituído das seguintes fases:

I) $\mathrm{MMI}=$ extensão de joelho e MMS=Extensão de braços alternados a frente; Flexão/extensão de cotovelos na horizontal; Flexão/extensão horizontal de ombros; Adução/abdução de ombros; Extensão de braços a frente.

II) $\mathrm{MMI}=$ flexão do quadril e MMS=Extensão de braços a frente e atrás; Adução de ombros alternado ao joelho oposto; Movimento da braçada do nado peito; Extensão de braços alternados a perna para baixo; Flexão/extensão horizontal de cotovelos alternado.

III)MMI=chute a frente/flexão do quadril e MMS=Flexão/extensão horizontal de ombros e adução/abdução de ombros; Adução das escápulas; Extensão de braços alternados a perna a frente; Extensão/flexão de cotovelos na vertical; Extensão/flexão de cotovelos na horizontal.

IV)MMI=flexão do quadril em abdução/perna $180^{\circ}$ e MMS=Movimentos de abraçar a água; Flexão/extensão horizontal de ombros com cotovelos flexionados em 90\%; Extensão de braços a frente e ao lado; Extensão alternada de braços contrários à perna; Abdução/adução de ombros posteriormente.

V) $\mathrm{MMI}=$ flexão de joelhos e MMS=Abdução/adução de ombros posterior alternadamente; Extensão de braços a frente; Flexão de cotovelo na vertical; Flexão de ombros; Adução/abdução de ombros.

VI) $\mathrm{MMI}=$ deslize frontal e MMS=Extensão alternada de braços a frente; Flexão/extensão de cotovelos na horizontal; Flexão/extensão de ombros; Flexão/extensão horizontal de ombros; Extensão de braços para baixo e para frente.

Durante o repouso $(5 \mathrm{~min})$ foram coletados os dados de $\mathrm{VO}_{2}$ (cada 20 segundos), de $\mathrm{FC}$ (cada
60 segundos) e de [Lac] (aos 5 minutos), a fim de constatar o estado de repouso.

Quanto à obtenção das amostras de sangue, 20 minutos antes das coletas fez-se assepsia no lóbulo da orelha e colocou-se uma fina camada da pomada vasodilatadora Finalgon. Após, retirou-se a pomada e perfurou-se o local com uma microlanceta descartável e realizou-se a coleta de sangue de repouso, ao final do período inicial, ao final de cada fase do período principal (350 segundos) e ao findar o período final da aula. As amostras foram coletadas em um capilar (EKF-Diagnostic), o qual foi preenchido completamente com sangue e colocado em um microtubo do tipo eppendorf (EKF-Diagnostic). A seguir, foram analisadas pelo lactímetro portátil Biosen 5030 (EKF-Diagnostic).

Para os dados de IPE, a Escala de Borg foi apresentada ao avaliado 30 segundos antes do final do período inicial, do final de cada fase do período principal e do término do período final. Já os dados de $\mathrm{VO}_{2}$ e de $\mathrm{FC}$ foram obtidos a cada 20 segundos durante a aula de hidroginástica.

O Gcal foi fornecido pelo próprio analisador de gases, estimado a partir dos resultados de $\mathrm{VO}_{2}$.

Para a análise dos dados, fez-se uso da estatística descritiva (média, desvio padrão, valores mínimos, máximos e percentuais) e ANOVA medidas repetidas, com o teste post hoc Student-Newman-Keuls, adotando-se como nível de significância 5\% (Pacote estatístico SPSS, versão 15.0).

\section{RESULTADOS}

As características do grupo de estudo são apresentadas na tabela 1 .

Ao observar o desvio padrão, pode-se perceber que os sujeitos apresentaram características distintas, evidenciados nos valores mínimos e máximos, retratando a realidade dos praticantes de hidroginástica, com características distintas realizando a mesma aula.

Os resultados da FC, do $\mathrm{VO}_{2}$ e da [Lac] durante os períodos da aula de hidroginástica são apresentados na tabela 2 .

Pode-se verificar que tanto a FC, quanto o $\mathrm{VO}_{2}$ apresentaram diferenças entre os períodos da aula. Já a [Lac] apresentou diferenças significativas apenas entre o período inicial e o principal e entre o inicial e final.

Os valores percentuais em relação aos resultados de $\mathrm{VO}_{2}$ pico e FCmáx obtidos no teste ergoespirométrico podem ser observados na tabela 3 . 
Tabela 1. Idade, massa corporal (MC), estatura, FC de repouso (FCR), pico do $\mathrm{VO}_{2}\left(\mathrm{VO}_{2}\right.$ pico) e percentual de gordura corporal (\%GC).

\begin{tabular}{lcccc}
\hline Variáveis & Média & Desvio Padrão & Mínimo & Máximo \\
\hline Idade (anos) & 23,06 & 2,05 & 20,00 & 27,00 \\
MC (kg) & 54,29 & 6,62 & 41,80 & 68,60 \\
\hline Estatura (cm) & 159,6 & 6,58 & 144,50 & 171,70 \\
FCR (bpm) & 78,59 & 7,71 & 66,00 & 89,00 \\
VO ${ }_{2}$ pico (ml/kg.min) & 48,83 & 7,3 & 37,10 & 64,00 \\
\%GC & 22,73 & 3,88 & 15,84 & 28,48 \\
\hline
\end{tabular}

Tabela 2. Resultados de FC, $\mathrm{VO}_{2}$ e [Lac] durante os diferentes períodos da aula de hidroginástica.

\begin{tabular}{|c|c|c|c|c|c|}
\hline \multirow[b]{2}{*}{ Variáveis } & \multicolumn{3}{|c|}{ Períodos da aula } & \multirow[b]{2}{*}{$\mathrm{F}$} & \multirow[b]{2}{*}{$p$} \\
\hline & Inicial & Principal & Final & & \\
\hline$\overline{\mathrm{FC}}(\mathrm{bpm})$ & $94,47 \pm 9,54^{\mathrm{a}}$ & $146,65 \pm 14,71^{b}$ & $109,56 \pm 11,73^{c}$ & 72,879 & 0,000 \\
\hline $\mathrm{VO}_{2}(\mathrm{ml} / \mathrm{kg} \cdot \mathrm{min})$. & $8,54 \pm 5,36^{a}$ & $23,22 \pm 3,77^{b}$ & $4,95 \pm 1,38^{c}$ & 93,864 & 0,000 \\
\hline $\mathrm{Lac}(\mathrm{mM})$ & $1,55 \pm 0,87^{\mathrm{a}}$ & $3,58 \pm 1,69^{b}$ & $2,88 \pm 1,54^{b}$ & 7,922 & 0,001 \\
\hline
\end{tabular}

Letras diferentes para a mesma variável representam diferença estatisticamente significativa.

Tabela 3. Resultados de $\mathrm{FC}$ e $\mathrm{VO}_{2}$ em termos percentuais relativos aos resultados máximos obtidos no teste ergoespirométrico.

\begin{tabular}{lccc}
\hline & \multicolumn{3}{c}{ Períodos da aula } \\
Variáveis & Inicial & Principal & Final \\
\hline $\mathrm{FC}$ & $47,04 \%$ & $76,16 \%$ & $58,48 \%$ \\
$\mathrm{VO}_{2}$ & $14,52 \%$ & $50,08 \%$ & $14,29 \%$ \\
\hline
\end{tabular}

De acordo com os resultados apresentados na tabela 3, é possível constatar que, em termos relativos, a resposta da FC foi maior que a resposta do $\mathrm{VO}_{2}$ em todos os períodos da aula.

Com relação ao IPE, verificou-se a mesma percepção para todas as avaliadas durante o período inicial e final da aula (IPE de 7 e 8 , respectivamente). Já durante o período principal, obteve-se como resposta média do grupo investigado um IPE de $11,20 \pm 1,15$, o que corresponde à percepção de um exercício caracterizado como razoavelmente fácil.

Na tabela 4, são apresentados os resultados de Gcal durante os diferentes períodos da aula e considerando o total da mesma, não se observando nenhum comportamento fora do esperado.

Tabela 4. Média do gasto calórico (Gcal) durante o período inicial, principal, final e o tempo total da aula de hidroginástica.

\begin{tabular}{lc}
\hline Períodos da aula & Gcal \\
\hline Inicial (kcal) & 10,01 \\
Principal (kcal) & 242,18 \\
Final (kcal) & 9,91 \\
Total (kcal) & 262,10 \\
Total (kcal/min) & 5,80 \\
\hline
\end{tabular}

\section{DISCUSSÃO}

O comportamento da FC durante a aula de hidroginástica apresentou alterações significativas dos resultados nos diferentes períodos da aula devido às mudanças na intensidade do esforço (Tabela 2). Esses resultados vão ao encontro da literatura ${ }^{7}$, que mostra que a intensidade de esforço é proporcional ao aumento da cadência, visto que os valores de FC durante a fase principal da aula (aeróbica) são maiores que os verificados na fase inicial (aquecimento e alongamento) e final (alongamentos).

O comportamento dessa variável durante exercícios acíclicos, no meio líquido ${ }^{17,18}$, mesmo utilizando métodos diferentes, apresentou-se semelhante. Vickery et al. ${ }^{17}$ mensuraram a FC durante exercícios aeróbios realizados no meio líquido e compararam com valores máximos obtidos em teste na esteira. Cada sujeito realizou 3 aulas pertencentes ao método Aqua Dynamics (20 minutos; 30 minutos e 60 minutos). A aula de 30 minutos é a mais semelhante à do presente estudo ( 35 minutos) e foi a que apresentou uma média da FC mais próxima do encontrado no presente estudo, tanto em termos absolutos ( $145 \pm 3 \mathrm{bpm}$ ), quanto em percentuais $(74 \pm 2 \%)$.

Eckerson e Anderson ${ }^{18}$ estudaram esta variável durante uma aula de ginástica aeróbica realizada na água e compararam com os resultados obtidos em teste máximo e encontraram uma média de $162 \pm 13 \mathrm{bpm}$ durante a fase aeróbia da aula, o que correspondeu a 82\% da FCmáx. Tais resultados são maiores que os apresentados nesse estudo $(76,16 \%)$ e que os estabelecidos por Vickery et al. ${ }^{17}$ que podem 
ser explicados pelo fato de que, em seu trabalho, foram realizados movimentos acima da linha do ombro, os quais podem afetar as respostas de $\mathrm{FC}^{9}$.

Mesmo quando a FCmáx foi predita ${ }^{19}$,os pesquisadores encontraram valores semelhantes do \%FCmáx (66\% a 70\% da FCmáx) ao do presente estudo.

Apesar de haver algumas diferenças metodológicas entre as aulas supracitadas ${ }^{17-19}$ e a relatada no presente estudo, constata-se que todas elas provocaram uma resposta adequada da FC, no que diz respeito à intensidade de trabalho para a melhora da aptidão cardiorrespiratória, visto que a faixa de intensidade recomendada pelo $\mathrm{ACSM}^{20}$ é de $65-90 \%$ da FCmáx.

Outra variável muito estudada é o $\mathrm{VO}_{2}$ já que permite avaliar o comportamento do sistema cardiorrespiratório. Sabe-se que o $\mathrm{VO}_{2}$ responde de forma linear à intensidade de trabalho ${ }^{21}$, o que foi constatado ao analisar os diferentes períodos da aula estudada, ou seja, com o aumento do esforço imposto (transição fase inicial-fase principal), o $\mathrm{VO}_{2}$ elevou-se de $14,52 \%(8,54 \mathrm{ml} / \mathrm{kg}$.min) para $50,08 \%(23,22 \mathrm{ml} / \mathrm{kg} \cdot \mathrm{min})$ do $\mathrm{VO}_{2}$ pico; e com a redução da carga de trabalho (transição fase principal-fase final), o $\mathrm{VO}_{2}$ diminuiu para $14,29 \%$ do $\mathrm{VO}_{2}$ pico $(4,95 \mathrm{ml} / \mathrm{kg}$.min).

Esses resultados corroboram os estabelecidos no estudo ${ }^{7}$ com exercícios isolados de hidroginástica nos quais o aumento da cadência (assim entendida como aumento de intensidade) também foi acompanhado por um aumento significativo do $\mathrm{VO}_{2}$. No entanto, assim como o aumento da cadência de execução, outros fatores podem alterar o comportamento do $\mathrm{VO}_{2}$, tais como, as diferentes áreas projetadas ${ }^{22}$ e a solicitação da massa muscular envolvida no movimento ${ }^{6}$. A aula de hidroginástica estudada utiliza uma maior mobilização de massas musculares durante o período principal, quando comparado aos movimentos do período inicial e final, apresentando, dessa forma, valores significativamente maiores neste período.

O $\mathrm{ACSM}^{20}$ indica que a faixa de intensidade recomendada para a melhora da aptidão cardiorrespiratória é de 50-85\% do $\mathrm{VO}_{2}$ máx. Ao observar a tabela 3, pode-se perceber que o $\% \mathrm{VO}_{2}$ pico apresentou-se dentro da faixa, na fase principal, verificando que o objetivo durante a fase principal da aula foi atingido. Esse resultado corrobora o estudo de Vickery et al. ${ }^{17}$ durante a aula de longa duração ( $51 \pm 2 \%$ do $\mathrm{VO}_{2}$ máx), a de média duração ( $57 \pm 3 \%$ do $\mathrm{VO}_{2}$ máx) e a de curta duração $(54 \pm 3 \%$ do $\mathrm{VO}_{2}$ máx), estando todos dentro da faixa reco- mendada. Diferenciando-se do estudo de Eckerson e Anderson ${ }^{18}$ (48\% do $\mathrm{VO}_{2}$ máx).

A diminuição dos valores de $\mathrm{VO}_{2}$, no meio líquido, pode ser justificado pela diminuição do peso hidrostático. Contudo, o aumento da resistência proporcionada pela densidade da água, que é maior que a do $\mathrm{ar}^{22}$, poderá ter favorecido o aumento do $\mathrm{VO}_{2}$.

Quanto ao IPE, as respostas obtidas do grupo investigado $(11,20 \pm 1,15)$ assemelham-se com os dados apresentados por Neves e Doimo ${ }^{19}$ (IPE entre 11 e 12), correspondendo à percepção de um exercício razoavelmente fácil. Os resultados de IPE também são semelhantes para mulheres $(11,44)$ que realizaram exercícios submáximos em esteira aquática a uma velocidade de $3,5 \mathrm{~km} / \mathrm{h}^{13}$.

Apesar do IPE durante a fase principal ter sido razoavelmente fácil, as respostas fisiológicas ocorridas em função da intensidade do esforço seriam suficientes para promover adaptações decorrentes de um treinamento aeróbico ${ }^{20}$. Assim, especula-se que o meio aquático proporciona uma melhora da condição física com uma percepção ao exercício razoavelmente fácil.

Já a importância do Gcal, diz respeito não apenas à manutenção da massa corporal, mas também à determinação da intensidade do exercício. Para Pollock et al. ${ }^{23}$,um Gcal total de 200 a $400 \mathrm{kcal}$ seria o recomendado para a melhora da capacidade de trabalho e o controle de peso em adultos saudáveis. A média do Gcal para toda a aula de hidroginástica aqui estudada foi de $262,10 \mathrm{kcal}$, enquadrando-se na faixa recomendada. Referindo-se ao Gcal por minuto, obteve-se como resultado 5,8, o que está de acordo com os trabalhos realizados $(5,7 \mathrm{kcal}$. $\min ^{18}$ e 5,9 - 6,5 kcal.min ${ }^{17}$ ). Os dados apresentados também se aproximam dos encontrados para a dança aeróbica. Em média, Weber ${ }^{10}$ e Igbanugo e Gutin ${ }^{11}$ registraram um Gcal de 6,2 e 6,57 kcal. min, respectivamente.

Fazendo referência à [Lac], constataram-se diferenças significativas quando foram comparados os valores obtidos no período inicial $(1,55 \mathrm{mM}) \mathrm{com}$ os obtidos no período principal $(3,58 \mathrm{mM})$ e entre o período inicial $(1,55 \mathrm{mM})$ e o final $(2,88 \mathrm{mM})$. Já entre o período principal e final não foi possível identificar diferença. Kindermann et al..$^{12}$ indicam que a [Lac] deve estar entre 2 e $4 \mathrm{mM}$ para o treino aeróbio. Os achados apresentados indicam que a carga de trabalho imposta no período principal da aula é suficiente para produzir alterações orgânicas $^{12}$. Contudo, no período final, eram esperados valores próximos de $2 \mathrm{mM}$. Moraes et al. ${ }^{2}$ ressaltam 
que após os exercícios de hidroginástica, a [Lac] demora em média 30 minutos para voltar à situação de repouso, justificando os valores encontrados.

\section{CONCLUSÃO}

Conclui-se que as variáveis fisiológicas estudadas e o IPE apresentam uma variação significativa em função das três fases da aula de hidroginástica, ou seja, aumentam (período inicial para o principal) e diminuem (período principal para o final) de acordo com a carga de trabalho imposta.

Além disso, constatou-se que a intensidade de esforço em termos percentuais, através das variáveis fisiológicas obtidas na fase principal da aula, estava dentro dos padrões estabelecidos pela literatura para exercícios aeróbios; permitindo inferir que a prática regular de hidroginástica com esta estrutura de aula, pode causar melhora da condição física de mulheres jovens com as características do grupo estudado.

\section{REFERÊNCIAS BIBLIOGRÁFICAS}

1. Kruel LFM. Alterações fisiológicas e biomecânicas em indivíduos praticando exercícios de hidroginástica dentro e fora d'água. [Tese de Doutorado - Programa de Pós-Graduação em Ciência do Movimento Humano]. Santa Maria (RS): Universidade Federal de Santa Maria; 2000.

2. Moraes EZC, Kruel LFM, Sampedro RMF, Lopes LFD. Metodologia de medida de esforço para exercícios de hidroginástica em diferentes profundidades de água. Kinesis 2002;27(1):43-186.

3. Arborelius M, Baldin, UI, Lilia B, Lundgren CEG. Hemodynamic changes in man during immersion with the head above water. Aerospace Med 1972;43(6):590-598.

4. Kruel LFM, Tartaruga LAP, Alberton CL, GraefF, Petkowicz $R$. Effects of hydrostatic weight on heart rate during immersion. Int J Aquat Res Educ 2009;3(2):178-185.

5. Pendergast DR, Lundgren CEG. The underwater environment: Cardiopulmonary, thermal, and energetic demands. J Appl Physiol 2009;106(1): 276-283.

6. Olkoski MM, Alberton CL, Pinto SS, Becker ME, Kruel LFM. Cardiorrespiratory responses of postmenopausal women to different water exercises. Int J Aquat Res Educ 2007;1(4):363-372.

7. Alberton CL, Coertjens M, Figueiredo PAP, Kruel LFM. Behavior of oxygen uptake in water exercise performed at different cadences in and out of water. Med Sci Sports Exerc 2005;37(5):S103.

8. Cassady SL, Nielsen DH. Cardiorespiratory Responses of Healthy Subjects to calisthenics Performed on Land Versus in Water. Phys Ther 1992;72(7):532-538.

9. Parker SB, Hurley BF, Hanlon DP, Vaccaro P. Failure of target heart rate to accurately monitor intensity during aerobic dance. Med Sci Sports Exerc 1989;21(1):230-234.
10. Weber $\mathrm{H}$. The energy expenditure of aerobic dancing. Fit Living 1974;8(1):26-30.

11. Igbanugo U, Gutin B. The energy cost of aerobic dancing. Res Q Exerc Sport 1978;49(3):308-315.

12. Kindermann W. The significance of the aerobicanaerobic transition for the determination of work load intensities during endurance training. Eur J Appl Physiol 1979;42(1):25-34.

13. Hall J, McDonald IA, Maddison PJ, O'hare JP. Cardiorespiratory responses to underwater treadmill walking in healthy females. Eur J Appl Physiol 1998;77(3):278-284.

14. Mader A, Liesen H, Heck H. Zur Beurteilung der sportartspezrfischen Ausdauerleistungfähigkeit. Sportarzt Ver Sportmed 1976(1);27:109-112.

15. Petroski EL, Pires N, Simões C. Validação de equações antropométricas para a estimativa da densidade corporal em mulheres. Rev Bras Ativ Física Saúde 1995;1(1):65-73.

16. McArdle WD, Magel JR, Lesmes GR, Pechar CS. Metabolic and cardiovascular adjustment to work in air and water at 18,25 and $33^{\circ} \mathrm{C}$. J Appl Physiol 1976;40(1):85-90.

17. Vickery SR, Cureton KJ, Langstaff JL. Heart rate and energy expenditure during aqua dynamics. Phys Sports Med 1983;11(3):67-73.

18. Eckerson J, Anderson T. Pysiological response to water aerobics. J Sports Med Phys Fitness 1992;32(3):255-261.

19. Neves ARM, Doimo LA. Avaliação da percepção subjetiva de esforço e da freqüência cardíaca em mulheres adultas durante aulas de hidroginástica. Rev Bras Cineantropom Desempenho Hum 2007;9(4):386-392.

20. ACSM. Manual de Pesquisa das Diretrizes do ACSM para Testes de Esforço e sua Prescrição. Rio de Janeiro: Guanabara Koogan; 2003.

21. Denadai BS. Consumo Máximo de Oxigênio: fatores determinantes e limitantes. Rev Bras Ativ Física Saúde 1995;1(1):85-94.

22. Pöyonen T, Keskinen Kl, Hautala A, Mälkiä E. Effects of aquatic resistance training on neuromuscular performance in healthy women. Med Sci Sports Exerc 2002;34(12):2103-2109.

23. Pollock ML, Wilmore JH, Fox SM. Health and Fitness Through Physical Activity. New York City: John Wiley and Sons; 1978.

\section{Endereço para correspondência}

Silvana Corrêa Matheus

Avenida João Machado Soares, no 1240, Bloco 3, Apartamento 313.

Bairro: Camobi.

CEP: 97110-000 - Santa Maria - RS/Brasil

E-mail: silvanamatheus@gmail.com 